\title{
Subject Index, Vol. 54, 1997
}

Acetaminophen 203, 285 Acetylcholine 261,312 Acetylsalicylic acid 49, 84 ai-Acid glycoprotein 271 Acid pump inhibitors 118 Actinomycin D 118 Activation-secretion coupling 153 Acute gastric mucosal lesions 203

- $\quad$ - phase proteins 271

Adenylate cyclase 144

Adipose tissue 169

$\alpha$-Adrenergic response 213

ß-Adrenergic response 213

B-Adrenoceptors agonist 144

Aggregation 261

ß2-Agonist activity 211

Airway hyperresponsiveness 261

Albumin 241,271

Alcohol 186

Aldose reductase inhibitors 76

Alkaline phosphatase 179

Allergen 2

Amiloride 241,256

Amitryptyline 312

-, acetylcholine-evoked contraction 314

-, endothelin 1-evoked contraction 314

Analgesia 92, 113

Analgesic effect, acetylsalicylic acid 84

Analgesics 285

Anesthetics 233

Angiotensin II 162

Anopheles mosquito 276

Antiasthma drugs 262

Antifibrogenic actions 179

Antiepileptic agents 113,319

- $\quad$-, plasma levels 319

Antigen challenge 2

Antihistaminic drugs 1

Anti-inflammatory drugs 285

Antimalarial drugs 276, 277

Antinociception 84

Antioxidant properties 58

Antioxidants 25

Antrum 305 
Aorta 24

Aortic lipid deposition 24

- $\quad$ rings 328

Aortocoronary bypass operations

162 Arachidonic acid 261

- metabolism 153

- metabolites 153 L-Arginine 43, 108, 298 Artemisinin compounds 276 Arterial blood pressure 108. 226 Articular cartilage 49 Aspartate 108

Assay, binding 86 -, DNA synthesis 51, 171 -, lipid peroxidation 59 -, PGE2 51 -, salicylate 86 -, serotonin 86 -, superoxide radicals 59 -, type-II collagen 51 Asthma 1,261

Atherosclerosis 24, 163,256 Atherosclerotic lesions 24 Atropine 64

Autonomic nervous system 233

Basophils 145 Behavioral profile 85 Bile duct ligation 179 Biliary excretion 276

- $\quad$ obstruction 179,180

Bilirubin 179

Binding sites 271

Biosynthesis, mucin 305

Blindness 256

B-Blockers 24

Blood flow 16,232

pressure $25,98,328$

- fall 232

transfusion 233 Bradycardia 211 Bradykinin 261

Brain 84,92,98, 113,232

cortex 108

stem 232

Bronchial asthma 1

- $\quad$ smooth muscle 144

Bronchoconstriction 2, 8, 261

Buspirone 187

Caffeine analogs 135 Calcium channel blocker 24

influx 211

ionophore 153

signal transduction 155

store 153 Cancer 92 Capsaicin 127 Captopril 241,256 Carbachol 298 Carbamazepine 113,319

Carbohydrate utilization 43 Carbon tetrachloride 43 Carboxylic acid 77 Cardiac events 163

index 18,225 Cardioprotective effects $25 \mathrm{Ca} 2+$ release channel 135 Carrageenan 285

Cartilage 49 Carvedilol 24

Cataract 256

- $\quad$ models 77

-, prevention 256

Cat, decerebrate 232

Caudate nucleus 92

CC14 179

Celiac artery, clamping 203

H4 Cells 169 
Cell damage 118

culture 119, 154, 171

viability 119 Cellular proliferation 169 Cerebral ischemia 108 Cerebrospinal fluid 92

Charybdotoxin 312 Chloride 33 Cholestasis 179 Chondrocytes, clusters 49 -, culture 49

Chronic liver disease 179 Cinitapride 193

KAR.GEK C1997S.Karger

Basel

335

E-Mail karger@karger.ch Fax+4 413061234 http://www. karger. ch

Circulation 16

Circular muscle strips 298

Citrulline 108

Clenbuterol 144

Cocaine 186

Collagen 50, 179

-, quantification 180

Colon 64

Colonic motility 64

- $\quad$ transit time 65

Congestive heart failure 25

Contractile response 298

Contractility 298

Contraction 312

Coronary artery disease 163,256

- $\quad$ atherosclerosis 162

Corpus mucosa 305

Cortex 108

Craniotomy 233

Cyclic AMP 144

Cyclooxygenase 84, 153

Cycloheximide 118

Cytochrome P450 276

Cytokine 153

-, treatment 155 Cytoprotection 57, 118

Damage 118 dbcAMP 144,305 Dexamethasone 285 Diabetes 169,241,256 Diabetic nephropathy 241 Diet 135

Dihydroalprenolol 211 Dihydroqinghaosu 276 Diuresis 127 Diuretic effect 33 DNA synthesis 49, 169 Drug metabolism 276

Edema 285 Eicosanoids 9 Electroshock seizure 320 Emptying 298 Endopeptide inhibitors 8

Endothelial function 163 Endothelin-1 312,328 Endotoxin 285 Enolicacid 49 Epilepsy 320

Equilibrium dialysis 272 Erosion 203

- $\quad$ area 57

Erythmea 285 Erythromycin 64 -, motor effect 64 Esophageal variceal hemorrhage

226 Essential hypertension 98,328 17ß-Estradiol 162 Estrogen 162

-, replacement therapy 162 Ethanol 118,193 
- $\quad$ - induced erosions 193

EukotrieneC4 145

Excitatory locomotive behavior

92 Excretion 33 -, sodium 127 Exercise-induced bronchoconstric-

tion 9 Extracellular matrix 179 Extrapyramidal system 92 Eye 256

Fat synthesis 169 Fecal pellet output 64 Fenoterol 144 Fibrosis 179 Fluid intake 187

Fluvoxamine 186 Foam cells 24 Formalin test 84, 86 Fructose-1,6-diphosphate 43

Galactose 76

Gastric contractility 298

emptying 298

mucosa 305

mucosal cells 118,119

- - damage 57,119,203

- contractility 298

- injury $57,119,203$

mucus secretion 193

- $\quad$ lesions, morphological and

histological observation 204

- $\quad$ ulcer 57, 193

Gastrointestinal toxicity 49

Gastroprotective properties 193

Gepirone 187

Glibenclamide 312

Globulins 271

Glomerular filtration 34,127 Glomerulosclerosis 242, 256 Glucose 241 Glutamate 108,320

$\gamma$-Glutamyltranspeptidase 179 Glutathione 92 Glyburide 169 Glyceryl nonivamide 127

Glycogen 43, 179 Glycolysis 43 Glycoprotein 193 Guinea pig 1, 8

Heart rate 16,98 Hemodynamics 16,225,233 Hepatic arterial blood flow 18

cell growth 169

drug metabolism 176 Hepatoma cells 169 Hepatoprotectors 179 Hexosamine 193 High-

affinity binding, melatonin

271 Hind paw 285 Histamine 9, 145, 153, 162,261,

305 -, mucin biosynthesis 308 Hot-plate 84,285 5-HT-receptors 84, 186 Human proteins 272

Hyaluronic acid 50 Hydrochlorothiazide 33,241 5-Hydroxyindole acetic acid 84 Hydroxyl

radicals 57, 203 5-Hydroxytryptamine 17, 193 Hyperalgesia 285, 286 Hypercholesterolemia

24 Hypercholesterolemic diet 24

- $\quad$ rat 24

Hyperglycemia 241,256 Hyperlipidemia 241 Hyperresponsiveness 262 Hypertension 25, 169, 225, 328 -, cadmium 328 Hypertrophy 169 Hyperventilation 8 Hypotension 232

IL-8 144

Immediate asthmatic response 1 Indole 113,194 Indometacin 118,285,298 Inflammation 285

Inflammatory cells 2, 145,261, 285

- reactions 261

Influx, myo-inositol 76 
Pharmacology Vol. 54, 1997

Subject Index

Insulin 169,241

- $\quad$ sensitivity 169

Interferon 179

Interleukin-1ß 144

Interleukin-4 153

Intracellular signal transduction

328 Intraocular pressure 211 Intrinsic sympathomimetic

activity 214 Ionophores 153 Ipratropium bromide 8 Ipsapirone 186 Ischemia 57, 108

- $\quad$ - reperfusion 57,203

Isoflurane 232

Isolated blood vessel 328

left atria 213

right atria 213

thoracic aorta 214

trachea 312

tracheal strips 214 Isometric tension 162 Isoproterenol 211

Ketanserin 16, 98 Kidney 33,98 -, function 37 -, lesions 251

Lamotrigine 319 Leminoprazole 118 Lens 76

Lesions, kidney 251 Leukocytes 57 Leukotriene D4 261

- $\quad$ - receptor antagonist 9

Leukotrienes 1

Lipid 241

-, deposition 24

-, levels 24

-, peroxidation 57, 203

- $\quad$ /water solubility 215

Lipoproteins 271

Lipoxygenase 153

5-Lipoxygenase inhibitor 9

Liver 43,169,179,277

-, $\quad$ damage 179

-, $\quad$ fibrosis 179

-, $\quad$ histology 171

-, $\quad$ homogenate 44

-, $\quad$ hypertrophy 169

-, $\quad$ isolated perfused 278

-, microsomes 278

- $\quad$ weight 169

Losartan 98

Low-molecular-weight protein

241 Lung 9 Lymphocytes 145

Macrolides 64 Macrophages 145 Mammary artery 162 Mast cells 153 Measurement, airway reaction

262 -, gastric emptying 299 
- $\quad$ hemodynamic 17,226

Melatonin 271

Meloxicam 49

Metabolic abnormalities 242, 256 Metabolism 276 -, mucin 305 Metastatic disease 92

Microalbuminuria 242 Micro injections 98 Microsomes 276 Microvascular leakage 2,262

Monocytes 24 Morphine 92,186,285 Motilin receptors 65 Motility 65 Mucin 118,305 -, biosynthesis 118 Mucosal damage 57,193 Mucus 193

gel 305

secretion 118 Muscle contraction 312

relaxants 312 Myocardial infarction 25, 162 Myoelectrical activity 64 Myo-inositol 76

$\mathrm{NaCl} 98$ Naloxone 84 Natriuresis 127 Nephropathy 241 NeurokininA 8 Neuronal membranes

320 Neuropathy 256 Neuropeptides 8 Neuroprotective efficacy 108 Neurotransmitter 320

Neutrophils 145

Nicotine 8, 186 Nifedipine 24, 64 Nitric oxide 298

- $\quad$ - synthase $43,108,298$

NO 43

Nociceptive protocols 287 Nociceptors 85 Non-adrenergic noncholinergic nerves 298 Nonsteroidal antiinflammatory

drugs 49, 285, 299 Noradrenaline 328 NO synthase blockade 298 NSAIDS 84

Oain tests 285 Obesity 169

Octanol/buffer partition coefficients 211 Ocular complications 256

- $\quad$ hypotensives 211

Omeprazole 120

Ondansetron 64

Opiates 84,92

Osmolar clearance 127

Oxidative stress 92

Oxygenase 50

Oxygen free radicals 108

Oxyntic region 305

PAF 1,261

- $\quad$ antagonist 1

Pain 85,92

Passive sensitization 155

Paw pressure 285

PBMCs 145

Pentylenetetrazole-evoked hind-limb extension 320

Perfused rat kidney 33

Peristalsis 64

Peripheral blood mononuclear cells 144

- resistance 18,225

Pharmacokinetics, lamotrigine

319 Phenylephrine 163, 211 Phorbol esters 153

- $\quad$ myristate acetate 153

Phosphodiesterases 145

Phosphoramidon 8 
Phorbol esters 153

Pineal gland hormone 271

Pioglitazone 169

Subject Index

Pharmacology Vol. 54, 1997

337

Plasma tryptophan concentration

113 Plasmodium berghei 276

falciparum 276

vivax 276 Platelets 1

- activating factor 1,153 ,

261

aggregation 261 Polyol formation 77 Portal blood inflow 17

hypertension 16,225

hypertensive rats 17,226

vein ligation 225

venous resistance 17 Postmenopausal women 162 Potassium 33, 127

chloride 162,328 Prazosin 16 Procaterol 144

Pro inflammatory mediators 153 Prokinetic agents 64 Propranolol 98, 144,211,225

Propulsive activity 64 Prostaglandin Ei 232 Proteases 153 Protein 241

binding 271

kinase isozymes 153

synthesis 118 Proteinuria 242 Proteoglycan 49 Proximal tubule 34 Pyrazinoylguanidine

241,256 Pyrilamine 1, 305

Quinazoline 17

Rabbit 64, 118,261 Ranitidine 305 Rats, diabetic 256 -, hypertensive 328 -, isolated trachea

312 -, portal hypertensive 225 -, STZ-diabetic 241 Receptor-binding experiments

214 Renal blood flow 18,233

clearance 33

disease 242

excretion 35,127

-, hydrochlorothiazide 37

failure 34, 127, 242, 243

sympathetic nerve activity 232,233

tolerability 50

- $\quad$ vascular resistance 18

Reperfusion 57, 203

Retinopathy 256

Ryanodine receptor 135

Sacroplasmic reticulum 135

Salicylate 84

Saltsensitive subjects 99

Sensitization 2

Serotonergic receptors 84,186 
Serotonin 16, 84, 113, 153, 162,

186,261 Serum binding 271 Signal transduction 153 Skeletal muscle 135, 169 Smooth muscle 64,298

- contraction 298 Sodium 33, 98

overload 98 Sorbinil 76 Spinal cord 84

superfusion 127 Spirohydantoins 77 Splanchnic blood 226 Stereotaxic brain injections 100

Stomach 57, 118

Stroke 25

STZrats 241,256

Substance P 8, 127

Sucralfate 57

Sulfhydryl compounds 193

Sulfidopeptide leukotrienes 9

Superoxide 57

- $\quad$ radicals 57,205

Survival 179

Sympathetic nerve activity 232

Tachycardia 98,211 Tachykinins 8 Tail flick 285 Taurocholate 118 Terazosin 98 Terbutaline 144 Tetragastrin 305 Tetrandrine 225 Tetrodotoxin 64 Thapsigargin 153 Theobromine 135

Theophylline 1, 144, 145 Thiazolidinedione 169

Thienodiazepine 261

Thiorphane 8

Thromboxane 1, 145

Thromboxane A2 1

Tirilazad 108

TNF- $\alpha$ production 145

Tonic contraction 211

Trachea 312

-, relaxation 211

Tracheal strips 211

Transport 76

Tricyclic antidepressants 312

Trimethaphan 108

Trinitroglycerin 232

Tryptophan 113

Tubular injury 34

Tumor necrosis factor 144

TXA2 1

- $\quad$ synthetase inhibitor 1

Ulcerative lesion 57 Ulcerogenic agents 118 Urinary excretion 241

- $\quad$ flow 33,127,241

Valproicacid 319 Vanidilol 211 Vanillin 211 Variceal bleeding 226 Vascular reactivity 162,328

resistance 226

smooth muscle 162,211

tissue 328 
tone 162,163,328 Vasodilating B-blockers 24 Vasodilators 17,24,232 Vasorelaxant activity 211 Viability 118

Voltage-sensitive sodium channels 320

Voluntary alcohol consumption 187

Women, postmenopausal 162

Xanthine 135

- derivatives 145

Zinc 58 ZNS 319 Zonisamide 319 Zopolrestat 76

338

Pharmacology Vol. 54, 1997 